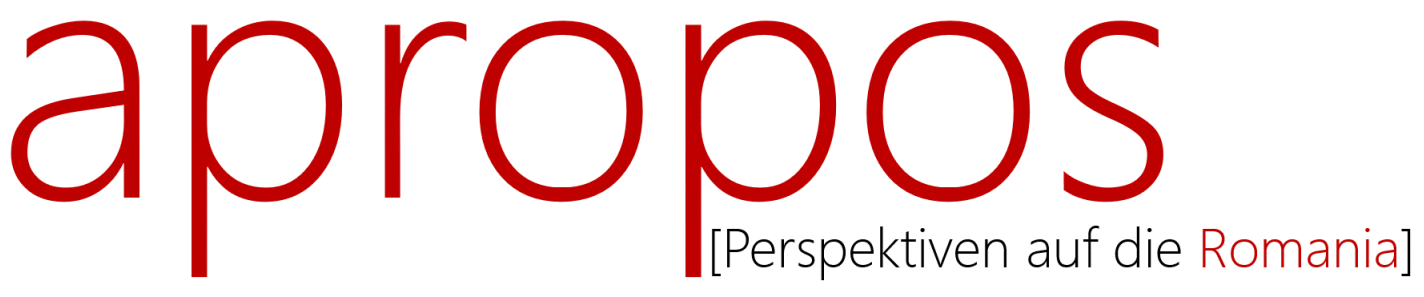

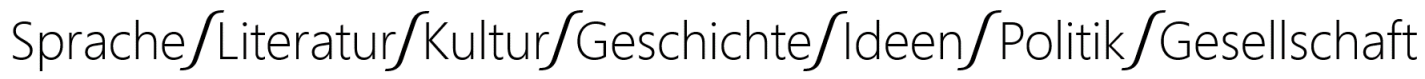

3 | 2019

Beziehungsweise(n)

Eine Frage der Ehre: Besitz und Geschlecht in der (spanischen) Literaturgeschichte

Nanette Rißler-Pipka

apropos [Perspektiven auf die Romania]

hosted by Hamburg University Press

2019, 3

STAATS- UND UNIVERSITÃTS

BIBLIOTHEK

H A M B UR G CARL VON OSSIETZKY

pp. 37-52.

ISSN: $2627-3446$

$\mathrm{DOl}$

https://doi.org/10.15460/apropos.3.1461

Zitierweise

Rißler-Pipka, Nanette. 2019. „Eine Frage der Ehre: Besitz und Geschlecht in der (spanischen) Literaturgeschichte", apropos [Perspektiven auf die Romania] 3, 3752. doi: 10.15460/apropos.3.1461

Except where otherwise noted, this article is licensed under a Creative Commons Attribution 4.0 International license (CC BY 4.0)

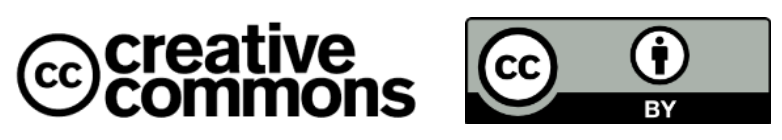


Nanette Rißler-Pipka

\title{
Eine Frage der Ehre \\ Geschlecht und Besitz in der (spanischen) Literaturgeschichte
}

\author{
Nanette Rißler-Pipka \\ ist habilitierte Romanistin und derzeit \\ stellvertretende Abteilungsleiterin \\ "Forschung \& Entwicklung" an der \\ Niedersächsischen Staats- und \\ Universitätsbibliothek Göttingen, \\ Programmleitung Digital Humanities \\ rissler-pipka@sub.uni-goettingen.de
}

Keywords

Ehre - Gabe - Drama - Gender - Besitz

\section{Ehre, Mythos, Religion und Geschlecht}

Bis in die 1970er Jahre hinein - denken wir nur an Heinrich Bölls Die verlorene Ehre der Katharina Blum (1974) - und in stark abgeschwächter Form auch noch bis heute ist ehrenhaftes Verhalten in heterosexuellen Geschlechterbeziehungen ausschließlich auf die Frauenehre bezogen. Diese sehr komplexen und sehr vage bis widersprüchlich beschriebenen Ehrkonzepte sind aus der Perspektive der Gender Studies Teil eines patriarchalischen Systems, das ein eigenes (männliches) starkes Selbst gegenüber einem abhängigen schwachen (weiblichen) Anderen definiert (vgl. dazu die bekannten Überlegungen von Kristeva, Irigaray, Cixous, Butler, u.a.). Die Dehnbarkeit des Ehrbegriffs erschwert das Sprechen und Verhandeln mit diesem auch in anderen Bereichen, wie Harald Weinrich am Beispiel des Nationalismus zeigen kann. Es geht auch im Kontext von Gabe, Ehre und Geschlecht um diskursive Macht und um verborgene Besitzverhältnisse, wie sich mit Mauss, Derrida, Foucault und Serres erläutern lässt. Das Beziehungs- und Besitzverhältnis der Geschlechter kommt aber auch in solchen (literarischen) Beispielen zum Vorschein, in denen Frauen gar nicht thematisiert werden, wie sich anhand von Baudelaires Prosagedicht La fausse monnaie (1869) oder auch Poes The Purloined Letter (1844) zeigen lässt.

Während sich der ehrenhafte Mann über die Ehre seiner Töchter, Frau und anderer weiblicher Familienmitglieder definiert und nur in zweiter Linie über ehrenhaftes Verhalten im Sinne von moralischem Verhalten, $d$. h. ehrlichem Sprechen und Handeln, bleibt dem weiblichen Teil der Bevölkerung nur die eigene Ehre, die sich konsequent über den eigenen Körper definiert. Schon allein die Begriffe wie 
„eigene" und „seine" deuten hier auf Besitzverhältnisse hin, die sich im Hintergrund des Ehrbegriffs verbergen.

Um diesen Aspekt des Ehrbegriffs von Geschlecht und Besitz näher zu beleuchten - ohne dabei in eindimensionale Argumentationen zu verfallen, die Ehrkonzepte als primitiv, barbarisch, und damit ,unchristlich' abstempeln, müssen wir auf die eigene ,westliche' (Literatur-)Geschichte blicken. Die spanische Literaturgeschichte, die auch eine europäische ist, zeigt hier Parallelen zwischen einem katholisch und im Nachhinein als, rückständig' betrachteten Ehrbegriff auf, vielleicht auch weil gerade in Bezug auf den Ehrbegriff Spanien vom Rest Europas gern zum kulturellen Fremdkörper erklärt wurde - ganz besonders im Zeitalter der Aufklärung.

Die Geschichte des Ehrbegriffs selbst unterscheidet auch zwei Stränge, den ersteren aus dem Tugend begriff abgeleiteten antiken Ehrbegriff, der nach Weinrich eher ein individueller, sozialer Wert ist, während der zweite dezidiert als "spanisch“ bezeichnete Ehrbegriff eine „Ethik der Öffentlichkeit" betrifft, von dem sich der Begriff des „Ehrenmanns" ableitet (Weinrich 1969, 224). Ehre ist hier ein Gut (Besitz), das verloren gehen kann. Wiedergewinnen kann es jedoch nur der Mann, indem er sich rächt. Rächende Frauen dagegen können ihre rein körperlich bestimmte Ehre auf diese Weise nicht wiederherstellen. Nur scheinbar regeln die Gesetze der Ehre den Handlungsspielraum der Personen so eindeutig wie in der griechischen Tragödie auf der einen Seite und der spanischen Comedia auf der anderen. Erst durch diese grundverschiedenen Theatertraditionen jedoch gelangen die beiden Ehrbegriffe hinaus in die Welt "und werden allgemeiner Kulturbesitz" (Weinrich 1969, 234). Beiden Traditionen gemeinsam ist allerdings die Figur des Deus ex machina bzw. eines als solchen handelnden Königs, der die vom Schicksal (antike Tragödie) oder von Ehrgesetzen (Comedia) gezwungenen und wehrlosen Menschen aus ihrer Notlage befreit. Beides bezeichnet Weinrich als "Mythos", den wir seit dem ausgehenden 19. Jahrhundert als solchen durchschaut hätten. Dies schließt eine Remythologisierung durch totalitäre Regime wie den Nationalsozialismus oder religiöse Fundamentalisten nicht aus (Weinrich 1969, 235).

Bezeichnend an Weinrichs Analyse ist, dass er dem als „privat“ markierten Ehrbegriff aus der antiken Tragödie einen spanischen - und nur im Nachgang auch europäischen - „öffentlichen“ Ehrbegriff gegenüberstellt. Da im zweiteren spanisch-europäischen Fall nicht das Individuum oder die Götter als Richter fungieren, sondern die "Anderen“, die Öffentlichkeit, reicht ein bloßer Verdacht für den Verlust der Ehre aus. Dieser Ehrverlust kann ebenso eine Beleidigung unter Männern sein wie die Untreue der Ehefrau oder die Entjungferung der unverheirateten Frau. In den letzteren Fällen bezieht sich der Ehrverlust aber weniger auf die Frauen als auf die Männer, denen diese zugeordnet sind, also Ehemann, Vater, Bruder, etc. Mit solchen Verdächtigungen und Intrigen lassen sich die schönsten Dramen schreiben wie ein Blick in die spanische Literaturgeschichte zeigt. Die Leitfrage dieser Studie ist, ob diese Dramen und andere literarische Beispiele das patriarchalische Ehrkonzept bestätigen oder aufdecken. Dass Letzteres im Sinne der Dekonstruktion auch die Machtverhältnisse offenlegt, muss 
durch eine entsprechende Lektüre gezeigt werden, ist aber schon immer im literarischen Text angelegt.

Foucault zeigt die diskursive Abhängigkeit der Geschlechter in Le Souci de Soi (L'Histoire de la sexualité, 3.). Er betrachtet wie Weinrich die antiken Verhältnisse und kommt zu dem Schluss:

On sait que l'adultère était juridiquement condamné et moralement blâmé au titre de l'injustice qui était faite par un homme à celui dont il détournait la femme. [...] c'est dire que la tromperie et le dommage était affaire entre les deux hommes. (Foucault 1984, 199)

Auch in den Texten der Antike geht es demnach im Sinne der individuellen Ehre nur um die Ehre der Männer. Die Frau ist dabei das Objekt der Ehre, kann das System daher nicht beeinflussen. Diese Konstellation einer "affaire entre les deux hommes" deutet bereits auf die aus Ehrkonzepten resultierende Duell-Praxis unter Ehrenmännern hin, die zwischen dem 16. und 19. Jahrhundert besonders in Frankreich (aber auch Spanien, Italien und anderen europäischen Ländern) unter dem Adel gepflegt wurde. Das Symbol der rauchenden Pistolen bei einem Duell wurde ebenso wie der Tabakrauch zu einem Symbol männlicher Identität, mit dem Derrida (auch in Bezug auf die eigene Meerschaumpfeife) im Dialog mit Mauss, Baudelaire und Poe in Donner le temps. La fausse monnaie (1991) spielt.

Die dahinterliegenden diskursiven Abhängigkeiten lassen sich in jeder Erzählung wieder und wieder aufdecken. Die bewusst ausgelassene oder stumme Frau macht in ihrer Abwesenheit oder fehlenden Handlungsoption umso mehr auf sich aufmerksam. Das Prinzip eines dahinterliegenden Ehrkonzepts, das auf eine Bestätigung männlich definierter Besitzverhältnisse abzielt, lässt sich von der Antike über die Bibel bis hin zur mannigfaltigen Ausgestaltung in westlicher Literatur nachweisen (vgl. dazu auch und kann keineswegs als Unterscheidungsmerkmal zwischen Orient und Okzident dienen.

\section{Susanna im Bade}

Die von der italienischen Barock-Malerin Artemisia Gentileschi in dem berühmten Bild Susanna e i vecchioni (Susanna und die Ältesten, 1610) gezeigte Geschichte der Susanna im Bade (Altes Testament, Buch Daniel 13,1-64) illustriert sehr gut den Ehrverlust durch üble Nachrede sowie die Diskursmacht auf Grundlage gesellschaftlicher Hierarchien. Der Ehrenkodex und ihre gesellschaftliche Position als Frau (Ehefrau) lassen der Protagonistin - ganz ähnlich wie später im Ehrendrama - keinerlei Handlungsoption: „Ich bin bedrängt von allen Seiten: Wenn ich es tue, so droht mir der Tod; tue ich es aber nicht, so werde ich euch nicht entrinnen" (Buch Daniel 13,23). Die erpresserische Forderung der Ältesten - „Sei uns zu Willen und gib dich uns hin! Weigerst du dich, dann bezeugen wir gegen dich, dass ein junger Mann bei dir war und dass du deshalb die Mädchen weggeschickt hast" (Buch Daniel 13,21-22) - funktioniert nur, weil der Verdacht eines Ehrverlusts bereits zur Verurteilung ausreicht und das Wort der Ältesten höher gewertet wird als das der Susanna. Sie entscheidet sich gegen das Hingeben und ist sich klar, dass sie damit ihr Leben gibt/opfert. In diesem ausweglosen Dilemma weiblicher Gabe, kann nur noch Gott selbst oder ein Abgesandter helfen. Im Ehrendrama würde hier 
die Figur des Deus ex machina eingreifen - in der Bibel ist es Daniel, der, als von Gott gesandter Held, die Ältesten der Falschaussage überführt und damit Susanna rettet. Ihre Ehre ist wiederhergestellt - und damit vor allem die Ehre ihres Ehemanns. Susanna hat richtig gehandelt mit ihrer Entscheidung, ihr Leben zu opfern. Doch ist sie nach wie vor das Objekt dieses Handels, da sie nur durch den göttlich-männlichen Helden gerettet wurde (vgl. Glancy 1993, 112 und 115). Gerade diese Konstellation legt die Geschichte offen und rechtfertigt die gesellschaftlichen Strukturen gleichzeitig. Denn Ungerechtigkeiten des Ehrkonzepts werden offenbar zuverlässig von Gott geradegerückt und stellen für das Funktionieren der Gesellschaft keine Gefahr dar. Wie die Literatur kann auch diese Bibelgeschichte als polyvalent gelten: Sie bestätigt auf der einen Seite ein ungerechtes Ehrgesetz, das Frauen zu Besitztümern macht, aber sie zeigt diese Ungerechtigkeit auch auf, macht sie explizit. Die Wahrheit entsteht hier wie dort in der Rede, dem Diskurs und nicht in dem, was wirklich passiert ist.

\section{Ehre, Gabe, Geschlecht und Wahnsinn}

Wenn wir nun davon ausgehen, dass die Ehre im Besitz des Patriarchen ist, der weibliche Familienmitglieder einschließt, können wir umgekehrt ebenso annehmen, dass die Gabe traditionell als weiblich definiert wird. Derrida stellt diese Annahme seiner Beschäftigung mit Mauss" „Essai sur le don“ in Donner le temps voran, indem er - wie so oft - von Heideggers Sein und Zeit ausgeht.

Et l'on pense alors à la dona, à la femme qui nous a requis depuis l'exergue, à toutes les questions de langue qui se croisent, en allemand et en français, dans les locutions « es gibt », et « ça donne ». (Derrida 1991, 37)

Auf sprachlicher Ebene werden „Frau“ und „Gabe" miteinander verbunden, wie z.B. „Leben geben“ oder das im Beispiel von Susanna im Bade bereits erwähnte "sich (hin-)geben" - und doch liegt in dieser Zuordnung schon ein Widerspruch. Denn kann die Frau geben, ohne zu besitzen? Wenn ja, was gibt sie dann? Derrida würde vermutlich antworten: "die Zeit“ („donner le temps"). Im Endeffekt ist die Frau diejenige, die gibt, die empfängt und auch Gegenstand der Gabe (Objekt) im Sinne des Frauentausches ist (die Tochter, Schwester zur Frau geben und eine andere dafür zur Frau nehmen, vgl. in Bezug auf Lévi-Strauss: Därmann 2010, 84).

Das Konzept der Gabe selbst ist ähnlich demjenigen der Ehre in sich nicht logisch, rational nachvollziehbar, sondern auf einer unmöglichen Bedingung aufbauend. Denn die Gabe kann in der christlich-westlichen Werte-Ordnung nur als reine Gabe gelten, wenn sie sich verschleiert. Dennoch erfordert die Gabe eine Wieder-Gabe, ohne dabei zum profanen Tausch zu werden. Das gelingt nur insofern der Beschenkte zwischenzeitlich vergisst oder vorgibt zu vergessen, dass die Gabe an sich erfolgte. Derrida betont auch hier die Rolle der Zeit: 
Condition pour qu'un don se donne, cet oubli doit être radical non seulement du côté du donataire mais d'abord, si on peut dire d'abord, du côté du donateur. [...] La simple conscience du don se renvoie aussitôt l'image gratifiante de la bonté ou de la générosité, de l'être-donnant qui, se sachant tel, se reconnaît circulairement, spéculairement, dans une sorte d'auto-reconnaissance, d'approbation de soi-même et de gratitude narcissique. (Derrida 1991, 38)

Auch das gebende Subjekt darf sich nicht in der Tugend des Gebens, der Großzügigkeit sonnen und sich der eigenen Tugend vergewissern, sondern muss dies ausblenden bis hin zum Vergessen. Nur durch die "Zeit" zwischen Gabe und Gegen-Gabe kann dies auch am viel zitierten Beispiel vom indianischen Brauch des Potlatch aus Mauss" „Essai sur le don" reibungslos funktionieren. Die Argumentation von Mauss liefert folgende Erklärung für die historischen Ereignisse: Stört der Weiße, der Kolonialherr dieses System von verschwenderischer Gabe und zeitlich versetzter Gegengabe durch eigene Gaben, führt dies zum Ruin unter den indianischen Stämmen in Nordwestamerika. Daher wurde der Potlatch in Reservaten Kanadas 1885 verboten (vgl. Därmann 2010, 56). Die von vielen Seiten französischer Theoretiker (Bataille, Derrida, Levi-Strauss, Serres) angebrachte Kritik an Mauss' Perspektive auf diesen indianischen Brauch, richtet sich auf die Erklärung der Gabe unter dem Gesichtspunkt des Tausches. Die Gabe wird hier effektiv zum Tausch und steht damit ganz im Zeichen der Ökonomie, wenn sie die Überbietung in der Gegengabe einfordert. Der Gewinn des Gebenden ist aber nicht nur die Hoffnung auf eine noch größere Gegengabe, sondern ein immaterieller Gewinn an Ehre und damit an sozialem Ansehen. Wie auch in den vorigen Beispielen der Antike und der Bibel stehen Ehre, Gabe und Besitz im engen Zusammenhang - unabhängig davon, ob es sich um monetären Besitz handelt oder um sozialen (im Sinne der Treue, der Ehefrau, Jungfräulichkeit, etc.).

Die Geste der Verschwendung wird hier wie dort zum dekonstruktiven Element, das die Grundsätze des Systems als irrational und widersprüchlich entlarvt. Die Ehre verschärft diesen Effekt.

C'est à propos de l'honneur que la folie fait irruption dans une scène qu'en vérité elle organise secrètement. C'est pour en venir à cette folie que nous nous sommes imposé ce détour. La folie qui s'insinue jusque dans le texte de Mauss, c'est un certain excès du don. II va peut-être jusqu'à brûler le sens même du don, menaçant au moins cette unité sémantique présumée qui autorise à parler encore de don. (Derrida 1991, 65-66).

Ehre ist in diesem Zusammenhang keineswegs ein Konzept zur Stärkung des patriarchalischen Systems, sondern fördert die Verschwendung, den Exzess, der in den Wahnsinn mündet und alle Ordnung zerstört.

\section{Babettes Fest}

Bevor wir auf die literarischen Beispiele eingehen, mit denen Derrida seine Thesen untermauert, lohnt sich für die Erläuterung genau jenes Zusammenhangs zwischen Ehre, Gabe, Geschlecht und Exzess (Verschwendung, die in den Wahnsinn mündet) ein Blick auf den Film Babettes Fest (Dänemark 1987, Originaltitel: Babettes gæstebud von Gabriel Axel nach einer Novelle von Karen Blixen). Die vor den Kriegswirren im Jahr 1871 aus Paris geflohene Star-Köchin Babette erhält in einem 
kleinen streng religiösen dänischen Dorf Asyl und wird Haushälterin zweier einsamer Schwestern, die sich beide aus Gründen der Entsagung in nonnenhafter Art aus dem Leben zurück gezogen haben. Als Babette im Lotto gewinnt, finanziert sie davon allerdings nicht die Rückkehr nach Paris, sondern verschwendet den gesamten Gewinn für ein Festmahl als Dank an die Dorfgemeinschaft. Den Gästen werden Verausgabung und Exzess nicht bewusst und sie können daher unwissend genießen. Diesen ohne Zweifel sehr körperlichen Genuss wollen wir nicht weiter interpretieren, aber es sei hier auf die Reaktion von Papst Franziskus verwiesen, der bekanntlich eher selten Kinofilme zitiert.

„Versag dir nicht das Glück des Tages [...] Beschenk den Bruder und gönne auch dir etwas" (Sir 14,14a.16a) [...] Man erinnere sich an die geglückte Szene in dem Film ,Babettes Fest', wo die großherzige Köchin eine dankerfüllte Umarmung und ein Lob empfängt: „Wie wirst du die Engel ergötzen!" Süß und belebend ist die Freude, anderen Vergnügen zu bereiten und zu sehen, wie sie genießen. (Papst Franziskus Amoris Laetitia, 112)

Nicht die Verführung der streng enthaltsamen Dorfbevölkerung wird hier in den Vordergrund gestellt, sondern die Ehre und Tugend der Gabe. Während es in der von Papst Franziskus zitierten Bibelstelle jedoch heißt "und gönne auch dir etwas", wird im Film klar deutlich, dass Babette sich ganz im Sinne der Enthaltsamkeit und weiblicher Selbstopferung der reinen Gabe verschreibt. Selbst isst sie nicht mit, sondern arbeitet mit perfekter Genauigkeit am Genuss der anderen. Nichtsdestotrotz wäre es in der Auslegung Batailles mit Hinblick auf Mauss“ "Essaie sur le don" eine "aktiv-selbstgestaltete Form der Verausgabung", die sich ähnlich wie die Sonne selbst verschwendet, verbrennt (vgl. Bataille in Därmann 2010, 65).

Bei aller argumentativen Einbettung der tugendhaften Handlung Babettes gelingt es auch der Amoris Laetitia des Papstes nicht, den Exzess, den Wahnsinn und den Genuss der Verausgabung ganz in vernünftige Bahnen zurück zu lenken. Der Genuss und Luxus des Essens verschwendet ebenso wie derjenige des Tabakrauchens (vgl. folgendes Kapitel und Derridas Donner le temps) die Gabe, die verschwindet, sich in einen anderen nicht-wertvollen Aggregatszustand verwandelt. Die Gegen-Gabe wird hier insofern nahegelegt wie im christlichen Kontext darauf verwiesen wird, dass Babette im Himmel die Engel ergötzen werde: ein recht eindeutiger Verweis auf den Lohn, den die Gebende, sich selbst opfernde Frau im Himmel erwartet. Im Diesseits jedoch kann damit die Geste der Verschwendung und des einbrechenden Wahnsinns nicht vollständig in die ökonomische Logik des Tausches aufgelöst werden. Diese Sicht auf Diesseits und Jenseits ist ebenso für das spanische Ehrendrama entscheidend (vgl. Kap. 3).

\section{Falschgeld und Tabakrauch bei Derrida und Baudelaire}

Die Tätigkeit des Rauchens ist vielleicht noch mehr als diejenige des verschwenderischen Essens dem Exzess und der sinnlosen Verausgabung unterstellt. Das Luxusgut des Tabaks löst sich im wahrsten Sinne des Wortes in Rauch auf und existiert nicht mehr. Aus heutiger Sicht vielleicht schwer nachvollziehbar, so hat dieser Genuss in vergangenen Jahrhunderten vor allem, 
aber nicht nur, Männerbünde zusammengeschweißt und geht auf indianische Riten wie die Friedenspfeife zurück, die auch beim Potlatch nicht fehlen darf.

Auch in Baudelaires für Derridas Studie titelgebender Erzählung La Fausse monnaie gehen die beiden Protagonisten (der Ich-Erzähler und sein Freund) rauchend eine Straße entlang, als einer der beiden einem Bettler ein Geldstück zuwirft und kurz danach zum anderen sagt: „C'était la pièce fausse." Diese kleine Bemerkung löst beim Ich-Erzähler eine Flut von Gedanken aus - letztlich die Geschichte selbst. Die Gabe - eine Handlung, um die eigene Ehre zu vermehren - im Sinne des Potlatch, aber auch im Sinne christlicher Moral - wird durch den Verweis auf den Betrug (das Falschgeld) vernichtet. Hätte der Freund diese Bemerkung nicht fallen lassen, wäre er möglicherweise im Ansehen und Ehre beim Ich-Erzähler und den Lesern gestiegen. So aber löst das vermeintliche Geständnis einen Konflikt zwischen den Freunden aus. Denn der Ich-Erzähler malt sich die Folgen des Falschgeldes aus: würde der Bettler am Ende ins Gefängnis kommen, wenn er versucht sich mit Falschgeld etwas zu essen zu kaufen? Aus dem Konflikt der Freunde um die Ehre könnte leicht eine Duellsituation entstehen.

Derrida betont in Bezug auf Baudelaires Prosagedicht zwei Punkte, die sich auf zwei Ebenen der Fiktion beziehen: erstens den Brief, den Baudelaire als Autor dem Text voranstellt und zweitens, den Inhalt der Geschichte selbst. Im Paratext, dem Brief Baudelaires, stellt der Autor seinen Text als „récit vrai“ heraus. Derrida merkt zu dieser komplexen Beziehung an: "Vraiment fictif, mais produit comme récit vrai par le narrateur fictif dans la fiction signée et forgée par Baudelaire" (Derrida 1991, 123). Ganz ähnlich wie Baudelaires Vorbild Edgar Allan Poe, scheint der Erzähler (und hier auch der Autor) den Wahrheitsgehalt betonen zu müssen, nur um am Ende genau dies bezweifeln zu können. Zusätzlich wird der Effekt der Verunsicherung erhöht, indem sich Baudelaire auf The Purloined Letter (Poe, 1844), bezieht. Die Wiederholung hebt die Einmaligkeit und Besonderheit der Gabe (die die Erzählung selbst ist) auf, macht sie zu Falschgeld, zerstört sie, verschwendet sie, löst sie in Rauch auf, wie der Tabak, den die beiden Protagonisten rauchen. Derrida spricht hier von einer „Duellsituation“, von der die Erzählung Baudelaires ausgehe:

Que le récit de La fausse monnaie procède à partir d'une situation apparemment duelle, qu'il ait liée avec un couple masculin, qu'il lie les deux partenaires mâles entre eux - et du tabac, c'est-à-dire à une consumation commune partant en fumée-, que cette fumée scelle aussi leur alliance, voilà un système de répétition, pour ne pas dire une compulsion. II cite et re-cite d'autres incipits narratifs, par exemple celui de La lettre volée de Poe. (Derrida 1991, 136)

Es sind die Inter- und Paratexte, die hier in der Wiederholung die Dekonstruktion einleiten. Denn mit der eingeführten Metaebene innerhalb der Geschichte selbst werden auch die Vor- und Randbedingungen der Ehre dem Zweifel der Fiktion ausgesetzt. Die Bemerkung über "la fausse monnaie" wird zum générateur d'art, wenn der Erzähler den Effekt als „prêtant des ailes à l'esprit“ (Baudelaire 1869) beschreibt.

Das Duell, der Tabak, die beiden Männerfreunde - wie schon Faucault bezüglich des Ehebruchs betont, ist es eine Sache zwischen zwei Männern. Hier scheint die 
Frau zu fehlen. Sie kommt in Baudelaires Prosagedichten nicht vor (Derrida bezieht sich auf La Fausse monnaie und Portraits de maîtresses) - und gerade daher bestimmt sie das Geschehen:

si la femme paraît absente de ce récit, son exclusion pourrait bien organiser la scène et en rythmer le temps comme une horloge. [...] Et les hommes parlent de la femme absente. La femme est leur sujet. (Derrida 1991, 134)

\section{Ehre im Intertext der spanischen, französischen und englischen Dramen}

Während Baudelaire und Poe im 19. Jahrhundert mit Ehre, Tabak, Betrug und Verschwendung im Wechselspiel der Ebenen von Fiktion spielen können, geht es im europäischen Ehrendrama zwischen dem 15. bis 18. Jahrhundert meist um blutige Tatsachen. Frauen und Männer stehen sich im Intrigenspiel gegenüber, die Ehre entscheidet über Existenzen und die Anderen, der öffentliche Schein, werden zum Richter - bis der König als Stellvertreter Gottes zur endgültigen Klärung eingreift. Es sind keine Gedankenspiele wie diejenigen der im Geiste berauschten Erzählerfiguren des 19. Jahrhunderts, aber die Grundlage des Dramas bildet doch Trug, Schein und Glaube bis hin zum Wahnsinn.

Dies beruht zum Teil auf gesellschaftlicher Realität. Im Laufe des Siglo de Oro führten Ehrkonzepte unter anderem zur Figur des verarmten Landadeligen, Hidalgo, der seinen Stand in fast absurder Weise über die Ehre aufrechterhält, auch wenn er sonst nichts mehr besitzt, wie uns die Episode zum Escudero aus dem Lazarillo de Tormes (1554) vorführt. Aus dem Stand der Hidalgos stammt auch der aus der Zeit gefallene Don Quijote (Cervantes 1605-15). Auch hier ist es der Schein, der engaño, der gegenüber einer kritischen Öffentlichkeit gespielt und aufrechterhalten werden muss.

Laut Weinrich entsteht daraus im Ehrendrama eine Dynamik zwischen Ehrverlust (oder dem Aufdecken, desengaño, des Ehrverlusts) und der Wiederherstellung der Ehre durch Rache oder Ähnliches: „die Ehre wird unter Blut und Tränen, oft auch unter Opferung des eigenen Lebens wiederhergestellt" (Weinrich 1969, 227). Gerade in diesem Wechselspiel von engaño und desengaño der spanischen Comedia werden Grenzen zwischen Wahnsinn und Vernunft verwischt und das Ehrkonzept verliert bereits seine Funktion einer Sicherung von patriarchalischen Verhältnissen.

Während der Burlador de Sevilla (Tirso de Molina, 1616) lange mit seinen Verstößen gegen die Ehre der betrogenen Ehemänner und Väter durchkommt, so reicht umgekehrt schon der bloße Verdacht des Ehrverlusts aus, um den Tod von Doña Mencía in El Médico de su honra (Calderón, 1637) zu verursachen. Nur scheinbar sind hier die Geschlechterrollen klar stereotypisch in Täter und Opfer eingeteilt. Die Kombination von Gabe und Ehre bedingt eine Reihe von Beziehungen, die ins Wanken geraten.

1. Die Gabe vermehrt Tugend und Ehre, kann aber auch mit Betrug und Falschgeld einher gehen. 
2. Liegt der Akzent auf dem Akt des Gebens, dann spielt die Währung (Falschgeld) keine Rolle. Die Performanz der Gabe funktioniert auch mit Lüge und Betrug - Gleiches gilt für die Ehre.

3. Gibt es eine ungleiche Beziehung zwischen demjenigen der gibt und demjenigen, der empfängt? Im Fall des Almosens ist per se keine Gegen-Gabe durch den Empfänger (sondern nur durch Gott im Jenseits) möglich. Ähnlich ungleichgewichtig ist die Beziehung zwischen männlicher und weiblicher Ehre - er holt sie sich gewaltsam durch Rache zurück, sie kann nur auf Gott hoffen.

\section{Unsichere Wahrnehmung bei Calderón und Shakespeare}

In den Ehrendramen des 16. bis 17. Jahrhunderts dient das angewendete Ehrkonzept eher als Folie, auf der sich die Intrige um Liebe und Betrug abspielt. Während in der klassischen Tragödie das Schicksal und die Götter die Helden zu den dramatischen Handlungen zwingen, sind es im Ehrendrama die übertriebenen gesellschaftlichen Konventionen, die in letzter Konsequenz angewendet zu absurden, dramatischen Verwicklungen führen. In seiner gattungsspezifischen Übertreibung ist das Drama besonders dazu geeignet, den Wahnsinn der Ehrkonzepte zu entlarven.

Auch wenn der Fokus bei der Betrachtung der Ehrendramen normalerweise auf den zentralen Konflikt gelenkt wird, fallen sowohl bei Calderón als auch bei Shakespeare die Hinweise auf eine möglicherweise unsichere Wahrnehmungssituation auf. Es ist nicht nur die burla, die uns als Zuschauer amüsiert, sondern auch die Unsicherheit der eigenen Wahrnehmung, die auf einen grundlegenden Betrug, auf ein engaño hindeutet, das uns von Baudelaire und Poe ebenso kunstvoll vorgeführt wird wie von Calderón in El Médico de su honra. Dies deutet auf einen Betrug, eine Unsicherheit hinsichtlich des Wahrheitsgehalts hin, von dem Derrida in Bezug auf La Fausse monnaie spricht. Der Betrogene befindet sich dann nicht nur als Protagonist im Drama, sondern auch in der Rolle der Zuschauer oder Leser.

Im Médico de su honra führt ein unglücklicher Zufall den verletzten Bruder des Königs, Don Enrique, zum Landhaus des Don Gutierre, der ausgerechnet mit Doña Mencía verheiratet ist, die in jungen Jahren vom Königsbruder umworben wurde dann aber standesgemäß Gutierre heiratete, um nicht ehrlos als Geliebte des príncipe zu enden. Enrique sieht, erwacht aus seiner Bewusstlosigkeit nun also Mencía vor sich und glaubt zu träumen:

ENRIQUE: $\quad \ldots$

estoy, si despierto sueño,

o si dormido oscurro,

pues un tiempo duermo y velo.

Pero ¿para qué averiguo,

poniendo a mayores riesgos

la verdad? Nunca despierte

si es verdad que ahora duermo;

y nunca duerma en mi vida

si es verdad que estoy despierto. (Calderón V. 164-171) 
Angekündigt durch die Aussprüche von Don Arias (seinem Diener) und Doña Mencía, „fuera sueño" (V. 100) "sueño fingido" (V. 94), umwirbt Enrique, gerade erwacht, Mencía ganz in höfischer Manier, wobei er die lang vermisste Angebetete für eine Traumerscheinung hält. Ohne Zweifel lässt sich die vielfältige Bedeutung des Traumes in der spanischen Comedia nicht mit den modernen Erzählerfiguren bei Poe und Baudelaire vergleichen, aber das Wechselspiel des engaño und desengaño nimmt dem Ehrendrama die Strenge und bittere Konsequenz, die gerade Calderón im Médico de su honra ausspielt.

Calderón bedient sich in fast unscheinbaren Kleinigkeiten bei Shakespeare, dessen unverkennbarer Humor gerade seine besonders blutigen Ehrendramen aufheitert. Dies scheint auch in El Médico de su honra aufgrund des blutigen Endes nötig. Kaltblütig lässt Gutierre seine Ehefrau durch einen beauftragten Arzt mithilfe des Aderlasses aus dem Weg räumen. Dabei ist er doppelt schuldig: Schließlich ließ er zuvor Leonor wegen unbewiesenen Verdachts der Untreue (vor der Hochzeit) sitzen, heiratete dann Mencía, die er wegen unbewiesenen Verdachts der Untreue umbringen lässt -, nur um dann frei zu sein für die Lösung des Königs: Gutierre muss Leonor heiraten, deren Ehre auf diese Weise wieder hergestellt ist. Es ist also keineswegs am Ende alles gut und die Ordnung wieder hergestellt. Nur im Lichte der Öffentlichkeit hat der König die Ehre der Leonor durch die Verheiratung mit Gutierre wieder hergestellt, der zuvor selbst seine Ehre durch den Tod der angeblich untreuen Ehefrau gerächt hat.

Obwohl nicht wirkliche Leidenschaften, sondern der bloße Anschein die entscheidende Rolle im Ehrendrama spielt, führt die Eröffnung Doña Mencías, sie sei verheiratet, dazu, dass Don Enrique die Flucht ergreifen will:

ENRIQUE:

Don Arias, dadme un caballo;

dadme un caballo, don Diego.

Salgamos presto de aquí

[...]

Estáse Troya ardiendo,

y Eneas de mis sentidos,

he de librarlos del fuego. (Calderón, V. 235-43)

Neben der Mythologie ist das eine klare Wiederholung der berühmten SterbeSzene aus Richard III: Bevor er allerdings den letzten Satz ruft „A Horse! A horse! My kingdom for a horse", formuliert er ganz ähnlich wie Enrique:

RICHARD:

Give me another horse, - bind up my wounds,

- Have mercy, Jesu! - Soft! I did but dream.-

O coward conscience, how dost thou afflict me!-

The lights burn blue. - It is now dead midnight.

Cold fearful drops stand on my trembling flesh.

What, do I fear myself? There's none else by:

Richard loves Richard; that is, I am I.

(Shakespeare, V. 3679-86) 
Bei Enrique ist es das schöne Ereignis, das Wiedersehen mit Mencía, das ein Traum sein muss, hier ist es das schreckliche, der nahende Tod, der ein Traum sein muss. Der Bösewicht Richard bekennt zum Schluss außerdem, dass er sich selbst liebt und er versichert sich seiner selbst: „I am I“.

In fast wortgleicher Formulierung sagt Doña Mencía: „Yo soy quien soy“ (Calderón, V. 133) - ein Ausspruch der prominent von Leo Spitzer 1947 als typisch für das spanische Ehrendrama und das spanische Drama an sich analysiert und erneut Shakespeare zugeordnet wurde. Spitzer nimmt Bezug auf die Kontingenz menschlicher Existenz im Gegensatz zur göttlichen und den noblen Wunsch, dem Ideal seiner Rolle zu entsprechen („,se representa el ideal que su personalidad debe aspirar" Spitzer 1947, 124). Das passende Zitat ist hier nicht aus Richard III, sondern aus Hamlet, wo der - laut Spitzer - größte Lügner des Stückes, Lord Polonius, sagt: "This above all: to thine ownself be true, And it must follow, as the night the day, Thou canst not then be false to any man" (Hamlet, Shakespeare, 1602, V. 564-66).

Wieder geht es um Ehrlichkeit, Ehre und Wahrheit, die aber gerade parodiert wird, weil die Zuschauer wissen, dass hier gelogen wird. Wichtig ist im Rahmen des spanischen Ehrendramas der soziale Stand und die damit verbundene Rolle: Mencía ist adliger Herkunft und verheiratet und damit ist ihr Handeln und ihr Schicksal bestimmt. Sie sagt es im Eingangs-Monolog, in dem sie ihren Tod voraus sieht und Gutierre, ihr Mörder, sagt es in seinem Monolog zum Wendepunkt des Stückes (Segunda Jornada), in dem er den Entschluss zum Mord fasst und sich mit den Zwängen der Ehre und der Gesellschaft entschuldigen kann: „Mencía es quien es y yo soy quien soy" (Calderón, V. 1649-50) - als gäbe es keine Alternative zu diesem Handeln. Denn es sind die von der Gesellschaft als übergeordnete Diskursmacht akzeptierten Ehr-Gesetze, die nur diese einzige Option, des Mordes einer Unschuldigen zulassen.

Genau das bezweifelt aber der Text selbst durch seine Struktur der Wiederholung und Verunsicherung von Wahrheit und Wahrnehmung.

Außerdem gibt es noch die Figur des Schelms, des gracioso, des Croquín, der auf die Frage des Königs antwortet, wer er sei: „soy quien vuestra majestad quisiere,“ (Calderón, V. 712-13), ich bin der, der ihr wollt, dass ich sei. Eine typische Antwort des Schelms, die humoristisch den Betrug, die Lüge und den Schein zum Gipfel führt. Auch hier ließe sich Shakespeare zum Vorbild erklären, man denke nur an As You Like It (1599), Twelfth Night or What you will (1599).

\section{Ehre und Gabe des Don Juan}

Während der männliche Täter, den wir leicht als Don Gutierre identifizieren können, in El Médico de su honra entschuldigt wird und der König auch die Ehre der betrogenen Doña Leonor wieder herstellen kann, liegt der Fall des Serientäters Don Juan etwas anders. Schon viel wurde über die Figur und den Mythos Don Juan auch aus Perspektive der Gender Studies - geschrieben. Wie kein anderer Charakter des Theaters führt Don Juan die Hypokrisie und die Fehler im Ehrenkonzept vor Augen. Daher war es auch naheliegend, dass der vermutlich größte Heuchelei-Detektiv der Theatergeschichte, Molière selbst, die spanische 
Vorlage mit Dom Juan ou le Festin de pierre (1665) verarbeitete. Genau diesem Stück widmet Michel Serres den Text „Le don de Dom Juan ou la nassaince de la comédie" (1968), den er später in seinem Buch Hermès (1969) wieder verwenden wird. Was gibt eigentlich Don Juan? Ist er nicht der nehmende Verführer? Nimmt er nicht den betrogenen Ehemännern, Vätern, Brüdern die Ehre und die Frauen weg und den Frauen...? Warum spricht Serres von einem „gebenden“ Don Juan?

Ähnlich wie Derrida am Beispiel Baudelaire die Beziehung der beiden Männer als vom Tabak gelenkte Duellsituation beschreibt, die Ehre und (Tabak)Gabe zusammenbringt, so verweist auch Serres auf freundschaftliche Gabe des Tabak, die uns Sganarelle, der Diener des Molière'schen Dom Juan erläutert:

Quoi que puisse dire Aristote et toute la Philosophie, il n'est rien d'égal au tabac: c'est la passion des honnêtes gens, et qui vit sans tabac n'est pas digne de vivre. Non seulement il réjouit et purge les cerveaux humains, mais encore il instruit les âmes à la vertu, et l'on apprend avec lui à devenir honnête homme. Ne voyez-vous pas bien, dès qu'on en prend, de quelle manière obligeante on en use avec tout le monde, et comme on est ravi d'en donner à droite et à gauche, partout où l'on se trouve ? On n'attend pas même qu'on en demande, et l'on court au-devant du souhait des gens; tant il est vrai que le tabac inspire des sentiments d'honneur et de vertu à tous ceux qui en prennent.

(Molière, $\mathrm{l} / 1,7$ )

Die Ehre der Gegen-Gabe wird hier angesprochen: Man bietet den Tabak an, bevor man gefragt wird und erhöht mit dieser Gabe die eigene Ehre. So wie diese Gabe und damit verbundene Ehre auf unausgesprochenen Erwartungen und ungeschriebenen Gesetzen besteht, so leicht ist es für den Molière'schen Dom Juan, sich diese offenen Verbindungen zwischen Ehre und Gabe zu Nutze zu machen.

Le secret ? Le voici : croisez sur la triple loi d'échange: ne rendez pas tabac pour tabac; c'est-à-dire bien pour bien, mot pour mot, amour pour amour, donnez au contraire mot pour bien et amour pour argent. Le créancier peut courir la campagne. (Serres 1968, 256)

Dom Juan gibt nicht Gleiches für Gleiches, sondern er gibt seine (wertlosen) Worte, auch implizite Ehrenworte (wie Eheversprechen) für Tabak, Liebe und Geld. Der schon von Derrida in Bezug auf Mauss angesprochene "crédit de l'honneur", der sich auf Tausch statt Gabe reduzieren lässt, wird hier sabotiert. Gerade diese Sabotage - ganz unabhängig von weiteren Gender-Implikationen im Don Juan Mythos - legt die Hypokrisie der Gesellschaft offen. Der Betrug gelingt Dom Juan wieder und wieder (auch im Sinne der von Derrida angesprochenen Wiederholung: Derrida 1991, 136, s.o. Kap. 2.2), weil er genau weiß, wie sich sein Gegenüber aufgrund der Ehrgesetze verhalten wird.

Dom Juan : Ce n'est pas moi qui rompt la promesse, c'est toi qui as failli à tes vœux... jusqu'à : ce n'est pas moi l'hypocrite, la société entière est l'imposture (Serres 1968, 261)

Ein schönes Beispiel aus Tirsos El Burlador de Sevilla ist die Verführung der Bauerntochter Aminta: Es reicht völlig aus, beim frisch gebackenen Ehemann von Aminta den Verdacht der Untreue seiner Frau aufkeimen zu lassen, damit dieser sich von ihr abwendet und sie als ehrlose Frau schutzlos Dom Juan überlässt. Ähnlich wie im Beispiel von Susanna im Bade (Kap. 1.1) gilt das Wort des 
Ehrenmanns Dom Juan schon aufgrund des gesellschaftlichen Standes mehr als dasjenige der unschuldigen Aminta. Dom Juan kann daher triumphierend und entlarvend bemerken: „Con el honor le vencí, porque siempre los villanos tienen su honor en las manos" (Tirso, Jornada Tercera, 100).

Don Juan täuscht und betrügt aber nicht nur beim Tausch, Gabe gegen Gabe, sondern auch bei der reinen Gabe, dem Almosen, das die Ehre des Gebenden erhöht wie wir es am Beispiel des Potlatch oder auch in La Fausse monnaie gesehen haben. Bei der Begegnung mit dem Bettler in Molières Dom Juan ou le Festin de pierre verwickelt er den Bettler in einen Tauschhandel (III/2) und verdreht auf diese Weise den Sinn des Almosens, der reinen Gabe. Dabei handelt es sich um einen ähnlichen Akt der Dekonstruktion wie wir ihn bei Baudelaire und dem falschen Almosen vorfinden. Dort ist es Falschgeld, hier ein erzwungener Tausch, Geld gegen Fluch, der den Sinn pervertiert und (in Rauch) auflöst. Der Bettler gehorcht trotz seiner existenziellen Sorge den Gesetzen der Ehre und der Tugend, indem er den Tausch (Geld gegen Fluch) ablehnt: „J'aime mieux mourir de faim“ (Molière, S. 56). Indem er auch dieses Gesetz der reinen Gabe und damit verbundener Ehre bricht, zeigt Don Juan auch hier die Heuchelei und patriarchalische Diskursmacht des Ehrkonzepts auf. Michel Serres bemerkt dazu: „Donner sans contrepartie; c'est se donner honneur et vertu, faire éclater sa puissance : cela s'appelle l'aumône." (Serres 1968, 254). Don Juan zeigt dagegen ebenso wie Derrida in seiner Baudelaire-Mauss-Lektüre, dass es die reine Gabe nicht gibt. Die Gabe besteht letztlich allein in der Zeit und, um mit Don Juan zu argumentieren, vielleicht noch in einem Wort, das ebenso gut eine Lüge (Falschgeld, engaño, burla sein kann).

\section{Rückblick und Ausblick mit Lorca}

Nicht erst im 19. Jahrhundert, wie Weinrich vermutet, wird demnach Ehre als Mythos durchschaut, sondern bereits zu Beginn des spanischen Ehrendramas im 17. Jahrhundert mit Tirsos Burlador de Sevilla und danach forciert von Molière im Dom Juan. Die vollständige Abhängigkeit von der Macht des Diskurses zeigt sich aber auch schon in Foucaults Beispielen aus der Antike und in der Geschichte von Susanna aus dem Alten Testament. Die Geschichten wiederholen sich und verweisen aufeinander. Es ist diese Wiederholung, die nach Derrida die Funktionsweise, Abhängigkeit und Machtverhältnisse des Diskurses von Ehre und Gabe aufzeigt.

Unter diesem Blickwinkel müssen wir auch die nach wie vor aus der heutigen Perspektive befremdlich wirkende Wiederholung der Ehrkonzepte des 17. Jahrhunderts in Lorcas Bauerntrilogie des 20. Jahrhunderts lesen. Fast unmöglich ist es, eine genaue Zeit zu bestimmen, zu der die Geschichten sich abspielen könnten, die Lorca in Bodas de sangre (1933), Yerma (1934) und La Casa de Bernarda Alba (1936) erzählt. Scheinbar hat sich in den Geschlechterverhältnissen und den Ehrkonzepten seit dem 17. Jahrhundert bis zum 20. Jahrhundert zumindest im ländlichen Teil Spaniens nichts verändert, beruht doch zumindest Bodas de Sangre auf einer wahren Begebenheit, die Lorca einem Zeitungsartikel entnahm (vgl. Grünnagel, Ueckmann, Febel 2016, 12 und Völpel 2014, 103). Schnell 
könnte man sich hier einer überkommenen Argumentation anschließen, die Spanien eine verpasste Aufklärung attestiert, obwohl dies lange widerlegt ist (vgl. Schlünder 2002, Tschilschke 2009).

Lorca gelingt es gleichzeitig die emotionale Not der unter dem Ehrenkodex leidenden Frauen und in den ambivalenten Mutterfiguren (MADRE und Bernarda Alba) den fatalen Geschlechtertausch zu zeigen, der das Patriarchat trotz fehlender Männer am Leben erhält. Die mit der Erziehungsaufgabe betrauten Mütter sind es, die ihre Söhne in die Blutrache treiben und ihre Töchter einsperren. Während die männlichen Protagonisten auf Nebenrollen degradiert werden, entwickelt sich das aus der Tradition geborene, ungerechte Diskursgefüge unter den Frauen weiter, die vor allem aus ökonomischen Gründen den Ehrenhandel weiter betreiben. Diese Ökonomie des Tausches verweist erneut auf Marcel Mauss und Derrida, wo der Ehrenhandel in den Überbietungswahnsinn der Gabe mündet. Der die Ordnung und Macht des Diskurses zerstörende Wahnsinn bricht bei Lorca durch das unkontrollierbare Element der Leidenschaft aus, die in der ausgehandelten Vernunftehe nicht vorgesehen ist. Die weiblich-gebende Mutterfigur entpuppt sich als eine zerstörerische Kraft, die entweder selbst an dem vorgegebenen Rollenbild zerbricht (Yerma) oder andere durch das starre Festhalten an Normen der Ehre ins Verderben stürzt (MADRE oder Bernarda Alba).

Nicht zu vergessen ist außerdem der historische Kontext, der trotz des Rückgriffs auf die Ehrkonzepte des 17. Jahrhunderts in Lorcas Tragödien einfließt. Während in Deutschland, Spanien und Italien seit Hitlers Machtergreifung 1933 Konzepte von Ehre und Männlichkeit benutzt werden, um den Mythos wiederzubeleben, legt Lorca das Dilemma und die Ungerechtigkeit solcher Konzepte offen. Ist das Spiel mit burla, engaño und desengaño bei Calderón und Tirso (und Molière) noch ein Vergnügen der Zuschauer, wird der öffentliche Schein einer willkürlich und körperlich definierten weiblichen Ehre (die immer auch diejenige des männlichen Verwandten ist) in Lorcas Tragödien zum alleinigen und gnadenlosen Richter über die Frauen.

Mit Lorcas Verbindungen zum Surrealismus, dessen Konzepte des Unheimlichen und Unerklärlichen sich auch in seiner Natursymbolik wiederfinden lassen, wird weiterer Zweifel an den Ehrkonzepten des Patriarchats gestreut. Auch wenn die Bezugnahme auf die als ,primitiv' gekennzeichneten Bräuche sowohl im Surrealismus als auch bei Lorca ambivalent gesehen werden können (vgl. z.B. aktuell Béhar 2019 und Assu et al. 2018), regt die Verunsicherung und Polyvalenz in der literarischen Darstellung zu einem Kreislauf der Dekonstruktion an.

\section{Bibliographie}

Assu, Sonny et al. 2018. „Histoire de l'art et potlatch : regards croisés entre la France et le Canada: Un débat virtuel entre Sonny Assu, Rémi Labrusse, Marie Mauzé et Charlotte Townsend-Gault, animé par Jean-Philippe Uzel“. Perspective 2 (31. Dezember 2018), 37-56. <https://doi.org/ 10.4000/perspective.11061>

BAUDELAIRE, Charles. 1869. "La Fausse monnaie", In CEuvres complètes de Charles Baudelaire, IV. Petits Poèmes en prose, Les Paradis artificiels, 8486, Paris: Michel Lévy frères.) <https://fr.wikisource.org/wiki/La_Fausse 


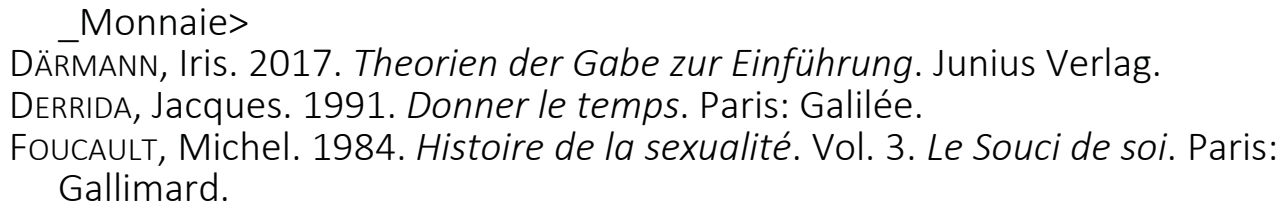

GARCíA LORCA, Federico. 2014 [1933]. Bodas de sangre: tragedia en tres actos y siete cuadros. Stuttgart: Reclam.

GARCíA LORCA, Federico. 2009 [1934]. Yerma: poema trágico en tres actos y seis cuadros. Stuttgart: Reclam.

GARCía LORCA, Federico. 2012 [1936]. La casa de Bernarda Alba: drama de mujeres en los pueblos de España. Stuttgart: Reclam.

GlanCY, Jennifer A. (1993). "The Accused: Susanna and Her Readers". Journal for the Study of the Old Testament, 18(58), 103-116. <https://doi.org/ 10.1177/030908929301805808>

GRünNAGEL, Christian, Natascha Ueckmann \& Gisela Febel (ed.). 2016. García Lorcas Drama "Bodas de sangre" und die Literaturtheorie: 17 Modellanalysen. Stuttgart: Reclam.

Moliere. 1994 [1665]. Dom Juan ou le festin de pierre. Paris: Editions mille et une nuits.

PAPST FRANZISKUS. 2016. Nachsynodales apostolisches Schreiben. Amoris Laetitia. Vatikan: Vatikanische Druckerei. <http://m.vatican.va/content/ francescomobile/de/apost_exhortations/documents/papa-francesco_ esortazione-ap_20160319_amoris-laetitia.html>

SCHLÜNDER, Susanne. 2002. Karnevaleske Körperwelten Francisco Goyas: zur Intermedialität der Caprichos. Tübingen: Stauffenburg.

SerReS, Michel. 1968. „Le Don de Dom Juan,“ Critique 205, 251-63.

SHAKESPEARE, William. [1592]. Richard III. [1600] Hamlet. <http://www. opensourceshakespeare.org/views/plays/plays.php>

TIRSO DE MOLINA. 1995 [1616]. El Burlador de Sevilla. Madrid: PML Ediciones.

TSCHILSCHKE, Christian v. 2009. Identität der Aufklärung/Aufklärung der Identität. Literatur und Identitätsdiskurs im Spanien des 18. Jahrhunderts, Frankfurt am Main: Vervuert

VöLPEL, Michael. 2014. „Nachwort“, in: García Lorca, Federico. 2014 [1933]. Bodas de sangre: tragedia en tres actos y siete cuadros,103-117, Stuttgart: Reclam.

WEINRICH, Harald. 1969. „Mythologie der Ehre.“ Merkur 23/251 (März 1969), 224-39.

\section{Zusammenfassung}

"Ehre" ist im Kontext religiös motivierter Auseinandersetzungen zwischen radikalisierten Fundamental-Islamisten und der westlich-christlichen Welt offenbar eine weiterhin schwierige Frage. Dabei glaubte man diese Dichotomie, die sich auf Orient und Okzident bezieht, in der öffentlichen Debatte überwunden zu haben. Ohne an dieser Stelle auf die Medienberichterstattung bezüglich sogenannter Ehrenmorde in westlichen Gesellschaften mit verschiedenen Migrationshintergründen eingehen zu können, noch die in Teilen problematische Haltung einer als ,zivilisiert' und ,westlich' definierten Gesellschaft gegenüber einer aufgrund ihrer Ehrkonzepte als ,primitiv' und ,barbarisch' definierten Gesellschaft im allgemeinen diskutieren zu können, fragt die vorliegende Studie mit Ansätzen aus Anthropologie, Philosophie und Gender Studies nach der Ehre-Beziehung der Geschlechter in einigen literarischen Beispielen vom 16.-17. Jahrhundert (Calderón, 
Tirso de Molina, Shakespeare, Molière) über das 19. Jahrhundert (Baudelaire, Edgar Allan Poe) bis zum 20. Jahrhundert (Lorca).

\begin{abstract}
In the context of religiously motivated conflicts between radicalized fundamental Islamists and the Western Christian world, "honour" remains a difficult question. At the same time the public believed to have overcome the dichotomy of Orient vs. Occident. Though, we cannot delve at this point into the media coverage of socalled honour killings in Western societies with different migration backgrounds, nor into the problematic attitude of a 'civilized' and 'western' defined society towards one that is defined as 'primitive' and 'barbaric' due to its concepts of honour. The present study explores the role of "honour" and gender relations in literary examples from the $16^{\text {th }}-17^{\text {th }}$ century (Calderón, Tirso de Molina, Shakespeare) to the 19th century (Baudelaire, Edgar Allan Poe) up to the 20th century (Lorca), relying on theoretical approaches from anthropology, philosophy and gender studies.
\end{abstract}

